University of Nebraska - Lincoln

DigitalCommons@University of Nebraska - Lincoln

October 1998

\title{
Target dependence of the triply differential cross section for low energy $(e, 2 e)$ processes
}

\author{
Cheng Pan \\ University of Nebraska - Lincoln \\ Anthony F. Starace \\ University of Nebraska-Lincoln, astarace1@unl.edu
}

Follow this and additional works at: https://digitalcommons.unl.edu/physicsstarace

Part of the Physics Commons

Pan, Cheng and Starace, Anthony F., "Target dependence of the triply differential cross section for low energy (e, 2e) processes" (1998). Anthony F. Starace Publications. 108.

https://digitalcommons.unl.edu/physicsstarace/108

This Article is brought to you for free and open access by the Research Papers in Physics and Astronomy at DigitalCommons@University of Nebraska - Lincoln. It has been accepted for inclusion in Anthony F. Starace Publications by an authorized administrator of DigitalCommons@University of Nebraska - Lincoln. 
Published in Many-body atomic physics: Lectures on the application of many-body theory to atomic physics, edited by $\mathrm{J}$.

J. Boyle and M. S. Pindzola. Cambridge University Press,

Cambridge/New York/Melbourne, 1998. Pages 261-286.

Copyright (c) 1998 Cambridge University Press.

Used by permission. 


\section{1 \\ Target dependence of the triply differential cross section for low energy $(e, 2 e)$ processes}

\section{Cheng Pan and Anthony F. Starace}

\subsection{Introduction}

In recent years, experimental studies on low and intermediate energy $(e, 2 e)$ processes have accumulated large amounts of triply differential cross section data. ${ }^{[428]-[439]}$ These $(e, 2 e)$ results, in which the energies and angles of both of the outgoing electrons produced in the electronimpact ionization process are specified, $[428,429]$ display strong electroncorrelation effects. Owing to the difficulty involved in describing precisely various electron correlations, in particular, the Coulomb interaction between the two final-state continuum electrons, only approximate theoretical treatments have been carried out. At present, theoretical understanding of these data and the underlying effects are far from complete. $[434,436,437]$

The near-threshold energy dependence of two electrons escaping from a positive ion has been studied theoretically by many authors using a number of methods. ${ }^{[440]-[451]}$ These studies cover the threshold behavior of the total and the differential cross sections for electron-impact ionization of atoms and ions.

In the early 1950 's, Wannier ${ }^{[440]}$ applied to this problem the idea that the near-threshold energy-dependence of a reaction could be derived by investigating only the long-range interactions among its final products, without having a detailed knowledge about a small "reaction zone," the size of which is of the order of magnitude of the Bohr radius. ${ }^{[441]} \mathrm{He}$ revealed the importance of the configuration $\mathbf{r}_{1}=-\mathbf{r}_{2}$ for the double escape of slow electrons from a positive ion by using methods of classical mechanics. He concluded that in the zero-energy limit all the orbits leading to the double escape approached asymptotically to this configuration. An assumption he made is that there is no strong selectivity against the two outgoing electrons emerging from the reaction zone in this configuration 
as compared to other configurations. He obtained a threshold law for the cross section for the ${ }^{1} S^{e}$ state of the two final-state continuum electrons. For neutral-atom targets, the electron-impact ionization cross section is predicted to vary as the 1.127 th power of the excess energy, which is the difference between the kinetic energy of the incident electron and the threshold energy for ionization.

Later, the double escape problem was treated by Temkin ${ }^{[442]}$ assuming that the dominant configurations leading to double escape are those with one relatively fast outgoing electron and one slow outgoing electron. Asymptotically, the corresponding processes can then be described as the relatively fast electron moving in the dipole field generated by the combination of the positive ion and the slow electron. A different threshold law was obtained which gives an oscillatory behavior very close to the threshold. [442]

The threshold behaviors of the energy partition and the angular distribution of the escaping electrons were studied by Vinkalns and Gailitis [443] using Wannier's technique. The energy partition close to threshold is predicted to be nearly uniform, and so for neutral-atom targets the differential cross section with respect to the energy of one of the two escaping electrons varies as the 0.127 th power of the excess energy. The angular distribution for cases in which the two escaping electrons leave in opposite directions is predicted to be independent of the excess energy $E_{\text {ex }}$. The distribution with respect to the mutual angle $\theta_{12}$ between the two escaping electrons is centered around $\theta_{12}=\pi$, and the width of this distribution is predicted to vary as $E_{\mathrm{ex}}^{1 / 4}$ for cases in which the charge of the residual ion is one or two.

The quantum mechanical formulation of the Wannier theory was first

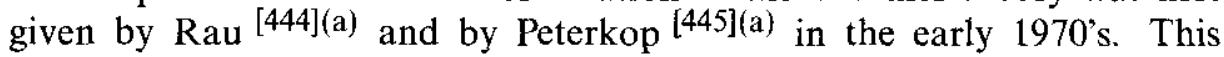
theory has been extended to treat all angular momentum states of the two escaping electrons. ${ }^{[446]}$ It has also been extended to treat the distribution of the angular momenta of the outgoing electrons, ${ }^{[447]}$ and it has been generalized to account for the finite mass of the ion and to include processes involving particles with various masses and charges. ${ }^{[448]}$ The Wannier theory and its extensions are often referred to as the WannierPeterkop-Rau (WPR) theory, and many experiments have been carried out to verify its predictions. ${ }^{[452]}$ Recent measurements for the spin asymmetry of electron-impact ionization of atoms have indicated the need to further extend the existing threshold theories. ${ }^{[453]}$

Earlier calculations for cross sections and other theoretical aspects of the electron-impact ionization problem have been reviewed by Rudge [454] and by Peterkop. ${ }^{[35]}$ Rudge pointed out that the methods used in actual calculations (up to 1968) did not take proper account of the Coulomb in- 
teraction between the two final-state outgoing electrons, and so they could not be expected to give precise cross sections and angular distributions for the outgoing electrons at low excess energies. Bottcher discussed various numerical methods for including the interaction between the outgoing electrons. ${ }^{[455]}$ Peterkop ${ }^{[456]}$ and Rudge and Seaton ${ }^{[457]}$ gave in the early 1960 's a relation specifying momentum-dependent effective charges which can be used to approximate the interaction between the two outgoing electrons, but calculations employing it were not reported until 1989. [458] The exact asymptotic boundary conditions for three charged particles interacting via Coulomb forces were first given in 1973, [343] but calculations which incorporated them also did not come until 1989. [344]

Measurements for the relative triply differential cross sections for $(e, 2 e)$ processes were first reported in 1969 by Ehrhardt et al. ${ }^{[459]}$ for the He atom and by Amaldi et al. ${ }^{[333]}$ for carbon. In 1972 and 1981, further measurements were made for $\mathrm{He}$ down to $6 \mathrm{eV}$ above the ionization threshold. ${ }^{[460]}$ In 1984 Fournier-Lagarde, Mazeau, and Huetz reported measurements for the triply differential cross section for He down to $1 \mathrm{eV}$ above threshold. ${ }^{[461]}$ To study the near-threshold experimental results, Altick used partial wave expansions and a correlation factor to describe the repulsion between the outgoing electrons. ${ }^{[462]}$ The resulting expressions fit the measured results very well. Crothers in $1986{ }^{[449]}$ reported an $a b$ initio calculation which explained well some of the characteristics of the measured near-threshold angular distributions even though only singlet states of the two outgoing electrons were included. He compared the contribution from the configurations emphasized in the WPR theory and the contribution from those emphasized in the theory of Temkin and found that the latter is relatively small. His results for the total and differential cross sections are consistent with the WPR theory.

Since 1987, a large number of $(e, 2 e)$ measurements have been reported for low excess energies [431]-[439]. A number of theoretical studies for the low-energy range have also been reported. [436],[439],[463]-[466] At present, there is no theoretical method which can reproduce the results of all the measurements.

Significant progress in treating the Coulomb interaction between the final-state continuum electrons has been made in the past several years by the use of wave functions which satisfy the asymptotic boundary conditions for three particles interacting via Coulomb forces. In 1989, Brauner, Briggs, and Klar first reported such a calculation for the triply differential cross section of the $\mathrm{H}$ atom. ${ }^{[344]}$ Franz and Altick have developed a partial-wave expansion for ionization amplitudes involving wave functions which satisfy the asymptotic boundary conditions. [351] Topics related to this progress are reviewed by P. L. Altick in chapter nine of this book. 
The main purpose of this chapter is to review our calculations, [464] whose aim is to explain the target dependence of the triply differential cross sections that has been observed in a number of recent experiments [430]-[432],[435],[438],[439] performed at relatively low energies above the ionization threshold for the two outgoing electrons leaving in opposite directions $\left(\theta_{12}=\pi\right)$. One reason for focusing on this $\theta_{12}=\pi$ geometry is that it is the most important geometry for near-threshold double-escape processes in the WPR theory. [440, 444, 445] Another reason is that up to now, except for $\mathrm{H}$ and $\mathrm{He}$ targets, the triply differential cross section for low-energy electron-impact ionization has been measured for $\mathrm{Ne}, \mathrm{Ar}, \mathrm{Kr}$, and Xe targets only in this geometry. ${ }^{[432,435]}$ In these experiments, for a given sharing of the excess energy by the two outgoing electrons, the differential cross section was measured, typically for a number of different angles between the incident electron beam and the vector connecting the two final-state outgoing electrons. When plotted against this angle, these measured results show striking target dependence.

Since at asymptotic separations the long-range fields in the final state of these ionization processes are the same, the observed target effects must be related to the short-range interactions between the incident electron and the target and between the final-state outgoing electrons and the residual ion. Therefore, in our calculations we focus mostly on the accurate treatment of such short-range interactions. For the interaction between the two final-state continuum electrons, we use an approximation which employs effective screening potentials ${ }^{[464](a, b)}$ because of the difficulty of treating simultaneously the long-range and the short-range interactions.

The methods we use are essentially distorted-wave methods, but we have also treated some electron-correlation effects perturbatively. Our calculations can be presented in the framework of many-body perturbation theory, and this is another purpose of the present chapter.

Theoretical studies for low-energy triply differential cross sections in various geometrical conditions other than $\theta_{12}=\pi$ have been reported by Altick, ${ }^{[462](a)}$ by Crothers, ${ }^{[449]}$ by Shaw and Altick, [462](b) by Selles, Huetz, and Mazeau, ${ }^{[430,463]}$ by Altick and Rösel, [462](c) by Brauner et al., ${ }^{[436]}$ by Jones, Madison, and Srivastava, ${ }^{[465]}$ by Botero and Macek, ${ }^{[466]}$ and by Jones and Madison, given in Ref. [439]. Interested readers are referred to these references for details of these studies.

In the next section our theoretical approach is discussed. Specifically, the partial-wave expansions for our approximate wave functions are given. Then, many-body perturbation theory is used to treat the matrix elements between $L S$-coupled wave functions. Finally, the formula for the differential cross section is reduced to a simple form for the $\theta_{12}=\pi$ case.

In a following section low-energy results of experimental and theoretical 
studies for the $\theta_{12}=\pi$ geometry are briefly reviewed. Calculated triply differential cross sections for $\mathrm{H}$ and $\mathrm{He}$ targets are compared with experimental results, and the observed difference between $\mathrm{H}$ and $\mathrm{He}$ is shown to stem from the short-range effects on the $s$-wave phase shifts of both incident and final-state continuum electrons. The comparisons between the calculated and the measured results for $\mathrm{Ne}, \mathrm{Ar}, \mathrm{Kr}$, and $\mathrm{Xe}$ are also given. Finally, the energy-dependence of the theoretical results for $\mathrm{He}$ is compared with available absolute experimental results.

\subsection{Theory}

Various aspects of the theory of electron-impact ionization of atoms and atomic ions were reviewed extensively by Rudge in $1968^{[454]}$ and by Peterkop in $1977 .{ }^{[335]}$ More recently, Brauner, Briggs, and Klar have discussed the use of wave functions that satisfy the asymptotic three-body boundary conditions; [344] Byron and Joachain have reviewed various higher-Born approximation methods; ${ }^{[467]}$ Curran, Whelan, and Walters have discussed a coupled-pseudostate method; ${ }^{[348]}$ Franz and Altick have given a partial-wave expansion for the asymptotic three-body boundary conditions; [351] and Jetzke, Zaremba, and Faisal ${ }^{[458]}$ have discussed the use of the effective charges specified by Peterkop ${ }^{[456]}$ and by Rudge and Seaton. [457]

Chapter 9 by P. L. Altick gives a review of recent progress on theoretical methods. Here we limit our discussion to the partial-wave expansions used for our approximate initial- and final-state wave functions, methods of many-body perturbation theory, and the simplified form of the formula for the triply differential cross section for the coplanar, $\theta_{12}=\pi$ geometry. In this work, we neglect relativistic effects and assume an infinite nuclear mass. Atomic units are used throughout this chapter except when otherwise indicated.

\subsubsection{Partial wave expansion}

The differential cross section for electron-impact ionization is given by

$$
\frac{d \sigma}{d \mathbf{k}_{1} d \mathbf{k}_{2}}=\frac{(2 \pi)^{4}}{k}\left|\left\langle\Psi_{f}^{-}|\Delta H| \Phi_{i}^{+}\right\rangle\right|^{2} \delta\left(E_{f}-E_{i}\right),
$$

or, equivalently, by

$$
\frac{d \sigma}{d \mathbf{k}_{1} d \mathbf{k}_{2}}=\frac{(2 \pi)^{4}}{k}\left|\left\langle\Phi_{f}^{-}|\Delta H| \Psi_{i}^{+}\right\rangle\right|^{2} \delta\left(E_{f}-E_{i}\right),
$$

according to formal scattering theory. ${ }^{[468]}$ In Eqs. (11.1) and (11.2), $k$ is the magnitude of the momentum of the incident electron, $\mathbf{k}_{1}$ and $\mathbf{k}_{2}$ are 
the momenta of the two continuum electrons in the final state, and $E_{i}$ and $E_{f}$ are the energies of the initial and final states. In Eq. (11.1), the final-state wave function $\Psi_{f}^{-}$is the exact solution of the full Hamiltonian, the initial-state wave function $\Phi_{i}^{+}$is the solution of an approximate Hamiltonian, and the perturbation $\Delta H$ is the difference between the exact Hamiltonian and the approximate Hamiltonian used to solve for $\Phi_{i}^{+}$. Similarly, in Eq. (11.2), the initial-state wave function $\Psi_{i}^{+}$is the exact solution of the full Hamiltonian, the final-state wave function $\Phi_{f}^{-}$ is the solution of an approximate Hamiltonian, and the perturbation $\Delta H$ is the difference between the exact Hamiltonian and the approximate Hamiltonian for $\Phi_{f}^{-}$. In Eqs. (11.1) and (11.2), the normalization for $\Psi_{i}^{+}$ and $\Phi_{i}^{+}$is assumed to be $\delta\left(\mathbf{k}^{\prime}-\mathbf{k}\right)$, the normalization for $\Psi_{f}^{-}$and $\Phi_{f}^{-}$is assumed to be $\delta\left(\mathbf{k}_{1}^{\prime}-\mathbf{k}_{1}\right) \delta\left(\mathbf{k}_{2}^{\prime}-\mathbf{k}_{2}\right)$, and the + and - superscripts on these wave functions denote, respectively, the outgoing-wave and incoming-wave boundary conditions.

In this chapter, Eq. (11.2) is used to calculate the triply differential cross section. ${ }^{*}$ Let $E_{\mathrm{ex}}$ denote the excess kinetic energy of the incident electron above the ionization threshold. Then $\delta\left(E_{f}-E_{i}\right)=\delta\left(\varepsilon_{1}+\varepsilon_{2}-E_{\mathrm{ex}}\right)$, where $\varepsilon_{j}=k_{j}^{2} / 2$ is the kinetic energy of the $j$ th final-state continuum electron. Noting that $d \mathbf{k}_{j}=k_{j} d \varepsilon_{j} d \Omega_{j}$ and integrating over $d \varepsilon_{2}$, Eq. (11.2) becomes

$$
\frac{d^{3} \sigma}{d \varepsilon_{1} d \overline{\Omega_{1} d \Omega_{2}}}=\frac{(2 \pi)^{4}}{k}\left|\left\langle\Phi_{f}^{-}|\Delta H| \Psi_{i}^{+}\right\rangle\right|^{2} k_{1} k_{2},
$$

where $k_{2}=\left(2 E_{\mathrm{ex}}-2 \varepsilon_{1}\right)^{1 / 2}$. In our calculations, we use a perturbation expansion to represent the initial-state wave function $\Psi_{i}^{+}$in Eq. (11.3). For the approximate final-state wave function $\Phi_{f}^{-}$, it is desirable to choose one that satisfies the asymptotic boundary conditions for three charged particles such that $\Delta H$ (i.e., the difference between the exact Hamiltonian and the approximate one satisfied by $\Phi_{f}^{-}$) is a short-range interaction. However, we use a more approximate final-state wave function which contains a product of two one-electron continuum wave functions. The interaction between the two final-state continuum electrons is approximated by using an effective screening potential for each of the continuum electrons. While this final-state wave function does not satisfy the exact boundary conditions, its form facilitates the use of partial wave expansions to treat the short-range interactions which govern the target dependence of the cross sections.

The initial-state wave function $\Psi_{i}^{+}$is characterized by the orbital and

\footnotetext{
- Equation (11.2) is used here for convenience in constructing a many-body perturbation expansion. The first-order calculation in this paper is the same as the one reported in Ref. [464], which employs Eq. (11.1) for the cross section.
} 
spin angular momentum of the $N$-electron target, denoted by $L_{0} M_{0} S_{0} M_{S_{0}}$, and by the momentum $\mathbf{k}$ and spin magnetic quantum number $m_{s}$ of the incident electron. The final state wave function $\Phi_{f}^{-}$is characterized by the orbital and spin angular momentum of the $(N-1)$-electron residual ion core, denoted by $L_{C} M_{C} S_{C} M_{S_{C}}$, and by the momenta, $\mathbf{k}_{1}$ and $\mathbf{k}_{2}$, and spin magnetic quantum numbers, $m_{s_{1}}$ and $m_{s_{2}}$, of the two continuum electrons.

A single-electron wave function characterized asymptotically by the momentum $\mathbf{k}$, and spin magnetic quantum number $m_{s}$ can be expanded as

$$
\phi_{\mathbf{k} m_{s}}^{( \pm)}(\mathbf{r})=k^{-1 / 2} \sum_{\ell=0}^{\infty} \sum_{m=-\ell}^{\ell} i^{\ell} e^{ \pm i\left(\delta_{\ell}^{C}+\delta_{\ell}\right)} Y_{\ell m}^{*}(\hat{\mathbf{k}}) u_{\varepsilon \ell m m_{s}}(\mathbf{r}),
$$

where the partial-wave states $u_{\varepsilon \ell m m_{s}}$ are given by

$$
u_{\varepsilon \ell m m_{s}}(\mathbf{r})=r^{-1} P_{\varepsilon \ell}(r) Y_{\ell m}(\hat{\mathbf{r}}) \chi_{m_{s}} .
$$

The $Y_{\ell m}$ functions in Eqs. (11.4) and (11.5) are spherical harmonics. In Eq. (11.5), $\chi_{m_{s}}$ is a two-component spinor, and the radial wave functions $P_{\varepsilon f}(r)$ are eigenstates of a single-particle Hamiltonian

$$
h_{l}=-\frac{1}{2} \frac{d^{2}}{d r^{2}}-\frac{Z}{r}+\frac{\ell(\ell+1)}{2 r^{2}}+V_{\ell}(r),
$$

where $Z$ is the nuclear charge and $V_{\ell}(r)$ is a radial potential. The function $P_{\ell \ell}(r)$ has the following asymptotic form

$$
P_{\varepsilon \ell}(r) \sim\left(\frac{2}{\pi k}\right)^{\frac{1}{2}} \sin \left[k r-\frac{\ell \pi}{2}+\frac{q}{k} \ln (2 k r)+\delta_{\ell}^{C}+\delta_{\ell}\right]
$$

as $r \rightarrow \infty$. In Eq. (11.7), $q$ is the net charge given by the asymptotic value of $Z-r V_{\ell}(r)$. In Eqs. (11.4) and (11.7), $\delta_{f}^{C}$ is the Coulomb phase shift, and $\delta_{\ell}$ is the non-Coulomb phase shift due to short-range interactions.

Using a partial-wave expansion such as that in Eq. (11.4) for the incident electron, we can couple each partial-wave function $u_{\varepsilon \ell m m_{s}}$ to the wave function of the target atom, forming antisymmetrized wave functions $\Psi_{i}\left(L_{T} M_{T} S_{T} M_{S_{T}}\right)$. The initial-state wave function $\Psi_{i}^{+}$for the target atom plus an incident electron with specified momentum can be expanded in terms of such $L S$-coupled states for the $(N+1)$-electron complex. Similarly, using partial wave expansions for the two final-state continuum electrons, we can couple each pair of partial-wave functions, $u_{\varepsilon_{1} t_{1} m_{1} m_{s_{1}}}$ and $u_{\varepsilon_{2} t_{2} m_{2} m_{s_{2}}}$, to form a two-electron function characterized by quantum numbers $L M S M_{S}$. This wave function can then be coupled to the wave function of the residual ion, forming antisymmetrized wave functions $\Phi_{f}\left(L_{T} M_{T} S_{T} M_{S_{T}}\right)$. The final-state wave function $\Phi_{f}^{-}$for the residual ion plus two continuum electrons, each with well-defined momentum, can be expanded in terms of such $L S$-coupled states. In what follows, we 
use unsuperscripted symbols $\Psi$ and $\Phi$ to denote these $L S$-coupled wave functions.

Then, the matrix element $\left\langle\Phi_{f}^{-}|\Delta H| \Psi_{i}^{+}\right\rangle$in Eq. (11.3) can be expanded in terms of the matrix elements of the $L S$-coupled states, $\left\langle\Phi_{f}|\Delta H| \Psi_{i}\right\rangle$.

\subsubsection{MBPT for $\left\langle\Phi_{f}|\Delta H| \Psi_{i}\right\rangle$}

Methods using many-body perturbation expansions for the optical potential [469]-[471] have been applied to electron-atom scattering problems, [469],[472]-[474] including the calculation of electron-impact ionization cross sections, ${ }^{[475]}$ by evaluating the imaginary part of the matrix element for the optical potential. Since our main concern here is the triply differential cross section for electron-impact ionization of atoms, we focus instead on the expansion of the matrix element $\left\langle\Phi_{f}|\Delta H| \Psi_{i}\right\rangle$.

To develop a perturbation expansion ${ }^{[476]}$ for the matrix element $\left\langle\Phi_{f}|\Delta H| \Psi_{i}\right\rangle$, we use the Hartree-Fock (HF) method ${ }^{[174]}$ to calculate the approximate final-state wave function $\Phi_{f}$ as well as the basis functions (orbitals) for expanding the initial-state wave function $\Psi_{i}$. Specifically, we use the self-consistent $\mathrm{HF}$ approximation for the wave function of the target atom with $N$ electrons. Thus we obtain as basis functions for the occupied one-electron states in the target atom the solutions of a set of single-particle Hamiltonians, each in the form of Eq. (11.6). For orbitals $(\varepsilon \ell)$ describing the incident continuum electron (as well as for excited orbitals), the general form ${ }^{[175]-[178]}$ of the single-particle potential in Eq. (11.6) is given by

$$
V_{\ell}=V_{g, \ell}+\left(1-\mathbf{P}_{\ell}\right)\left(V_{\varepsilon \ell}-V_{g, \ell}\right)\left(1-\mathbf{P}_{\ell}\right),
$$

where $\mathbf{P}_{\ell}=\sum_{i}\left|n_{i} \ell\right\rangle\left\langle n_{i} \ell\right|$, with the summation running over the occupied states of the target, and where $V_{g, \ell}$ is the potential for the occupied states. The right hand side of Eq. (11.8) reduces to $V_{\ell \ell}$ when there is no occupied one-electron state with the same $\ell$ in the target atom. Calculating occupied and excited states of a given angular momentum quantum number $\ell$ in the same Hermitian potential guarantees orthogonality.

For each partial wave $(\varepsilon \ell)$ of the incident electron, $V_{\varepsilon \ell}$ is derived according to ${ }^{\dagger}$

$$
\delta\left\langle\Phi_{i}|H| \Phi_{i}\right\rangle=0
$$

by keeping those terms with the factor $\delta P_{\varepsilon \ell}$. Using the HF wave functions for the target atom and for each partial wave of the incident electron, we can construct a zeroth-order initial-state wave function $\Phi_{i}$ corresponding

\footnotetext{
${ }^{\dagger}$ In Eq. (A2) of Ref. [464](b), $\Psi_{f}$ and $\Psi_{i}$ should both be $\Psi_{i, f}$. The subscripts were mistyped.
} 
to a zeroth-order Hamiltonian which is a sum of $N+1$ single-particle Hamiltonians.

We can define then the approximate final-state wave function $\Phi_{f}$. For the one-electron states in the residual ion, we use the basis functions obtained from the self-consistent solution for the initial-state target atom. For each one-electron partial wave $\left(\varepsilon_{j} \ell_{j}, j=1,2\right)$ of the two final-state continuum electrons, a HF potential $V_{\varepsilon_{j} \ell_{j}}$ is derived by neglecting the radial integrals involving four continuum orbitals in the matrix element $\left\langle\Phi_{f}|H| \Phi_{f}\right\rangle$ and by keeping those terms with the factor $\delta P_{\varepsilon_{j} \ell_{j}}$ in the equation

$$
\delta\left\langle\Phi_{f}|H| \Phi_{f}\right\rangle=0 .
$$

The potential derived this way does not include the interaction between the two continuum electrons in the final state, but we approximate this interaction by adding to this potential an effective screening potential. ${ }^{[464](a, b)}$ We use the relation derived by Peterkop ${ }^{[456]}$ and by Rudge and Seaton ${ }^{[457]}$ for the effective charges of the screening potential at large distances,

$$
-\frac{Z_{T}-\Delta_{1}}{k_{1}}-\frac{Z_{T}-\Delta_{2}}{k_{2}}=-\frac{Z_{T}}{k_{1}}-\frac{Z_{T}}{k_{2}}+\frac{1}{\left|\mathbf{k}_{2}-\mathbf{k}_{1}\right|},
$$

where $Z_{T}$ is the net charge of the residual ion, and $\Delta_{1}$ and $\Delta_{2}$ are the effective charges. For the configuration considered here in which $\hat{\mathbf{k}}_{1}=-\hat{\mathbf{k}}_{2}$, Eq. (11.11) can be satisfied by using the following effective charges: ${ }^{[458]}$

$$
\Delta_{i}=\frac{k_{i}^{2}}{\left(k_{1}+k_{2}\right)^{2}}, \quad(i=1,2) .
$$

Here also, Eq. (11.8) is used to ensure the orthogonality between each of the partial waves with the occupied one-electron states in the target atom having the same orbital angular momentum $\ell$. Since, for a given $\ell$, the one-electron partial wave for the two outgoing electrons in the final state and that for the incident electron are calculated using different potentials, they are not orthogonal to each other, although they are both orthogonal to the occupied state with the same $\ell$ in the target atom.

The difference between the exact Hamiltonian and the approximate Hamiltonian for the final state is

$$
\Delta H=\sum_{i<j=1}^{N+1} \frac{1}{\left|\mathbf{r}_{i}-\mathbf{r}_{j}\right|}-\sum_{j=1}^{N+1} V_{\ell_{j}}\left(r_{j}\right) .
$$

The difference between the exact Hamiltonian and the zeroth-order Hamiltonian for the initial state is also given by Eq. (11.13) for the corresponding single-particle HF potentials $V_{\ell_{j}}$. This latter difference, 


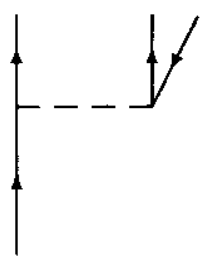

(a)

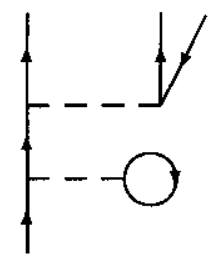

( b )

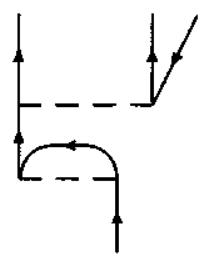

(c)

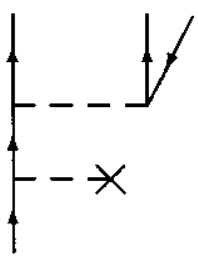

(d)

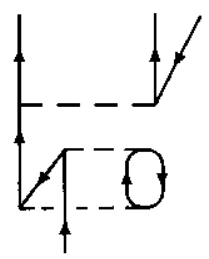

(e)

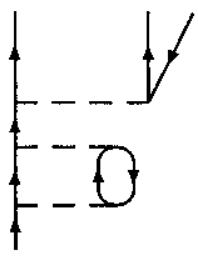

( f )

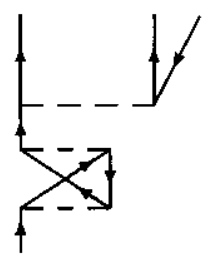

( g )

Fig. 11.1. Some diagrams contributing to the matrix element $\left\langle\Phi_{f}|\Delta H| \Psi_{i}\right\rangle$. Higher-order diagrams $(\mathrm{b})-(\mathrm{g})$ are treated by defining an appropriate potential for the radial wave function of the incident electron. Diagrams (f) and (g) can only be approximately included by using Hermitian potentials.

$\Delta H$, is treated as a perturbation for expanding the initial state wave function $\Psi_{i}$.

A perturbation expansion for the matrix element $\left\langle\Phi_{f}|\Delta H| \Psi_{i}\right\rangle$ can be obtained when the initial-state wave function $\Psi_{i}$ is expanded, and terms of this perturbation expansion can be represented by many-body diagrams. Examples of the diagrams are shown in Figs. $11.1-11.3$. In these diagrams, each horizontal dashed line with both ends connected to lines with arrows represents the Coulomb interaction term in Eq. (11.13), and each dashed line with a cross at one end denotes the interaction with the negative of the potential term in Eq. (11.13). Lines with arrows drawn downward represent vacancies (holes) in the ground-state target atom, and lines with arrows drawn upward denote continuum or bound excitations (particles). Time runs from bottom to top in these diagrams, and the order of a diagram refers to the number of horizontal dashed lines (interactions) in it. The open particle line on the bottom of each diagram represents the zeroth-order initial-state wave function, and the open hole line and the two open particle lines on top of each diagram denote the approximate final-state wave function. Notice that we do not include diagrams in which the two open particle lines are connected to two other particle lines at the ends of one Coulomb interaction line; these 
continuum-continuum interactions are considered to be accounted for by the effective screening potentials which are added to the potentials for the orbitals represented by the two open particle lines on top of each diagram.

Shown in Fig. 11.1 are some diagrams that we treat, either exactly or approximately, by using different potentials for the incident electron. The diagram of Fig. 11.1(a) gives the first-order matrix element. The diagram of Fig. 11.1(b) includes the correction to the first-order result due to the direct interaction between the incident electron and the bound electrons in the target atom, and the diagram of Fig. 11.1(c) includes the correction due to the corresponding exchange interaction. The diagram of Fig. 11.1(d) includes the correction due to the interaction with the negative of the single-particle potential used for calculating the wave function for the incident electron. When we use the HF potential derived using Eq. (11.9) for the wave function of the incident electron, the three diagrams in Figs. 11.1(b)-11.1(d) sum to zero. If we neglect the exchange terms in the HF potential for the incident electron, then only the diagrams of Figs. 11.1(b) and 11.1(d) sum to zero. The diagram of Fig. 11.1(e) can be treated by adding second-order terms to the HF potential used for the incident electron. The diagrams of Figs. 11.1(f) and 11.1 $(\mathrm{g})$ describe effects due to the interactions between the elastic scattering channel and inelastic scattering channels including impact ionization channels, and each of them comprises both real and imaginary parts. A complete treatment of such inter-channel interactions is in general difficult. However, the effects of these interactions on the elastic scattering channel can be estimated by calculating a second-order matrix element, which contains both a real part and an imaginary part. ${ }^{[475]}$ For the $\mathrm{He}$ target and for the incident energies of interest here, the imaginary part is small relative to the real part. So, in this work we neglect the imaginary parts of the diagrams of Figs. 11.1(f) and 11.1(g) and treat the real parts of these diagrams by adding second-order terms to the HF potential used for the incident electron. The second-order potential terms that we use to treat Figs. 11.1(e)-11.1(g) are dependent on the incident energy.

Shown in Fig. 11.2 are diagrams which describe the effects of the interactions of each final-state continuum electron with the bound electrons in the residual ion and with the negatives of the single-particle potentials used for calculating the wave functions of the continuum electrons. When we use the potentials derived according to Eq. (11.10) for the final-state continuum electrons, all the diagrams in Fig. 11.2 sum to zero for targets having closed-subshell residual ions. In general, inter-channel interactions between final-state wave functions with different intermediate angular momentum couplings are nonzero, so that 


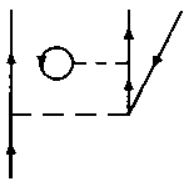

(a)

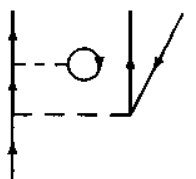

(f)

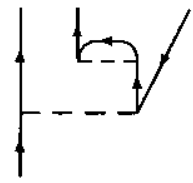

( $\mathrm{b}$ )

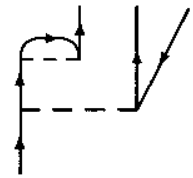

( $\mathrm{g}$ )

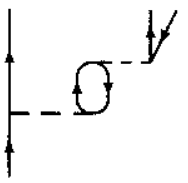

(c)

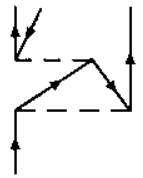

( h )

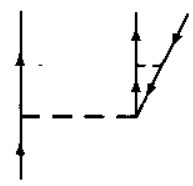

(d)

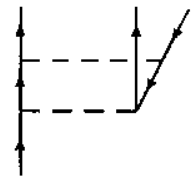

( i )

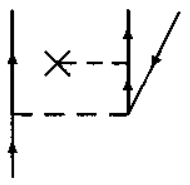

(e)

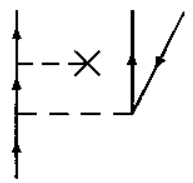

( j )

Fig. 11.2. Some second-order diagrams contributing to $\left\langle\Phi_{f}|\Delta H| \Psi_{i}\right\rangle$. For target atoms having closed-subshell residual ions, these diagrams sum to zero when potentials (of Hartree-Fock type) derived according to Eq. (11.10) are used for the radial wave functions of the outgoing electrons in the final state.

not all the diagrams in Fig. 11.2 can be cancelled by simply choosing appropriate single-particle potentials. To include inter-channel interactions for the case of helium targets, we use two-channel close-coupling solutions ${ }^{[477]}$ for the one-electron continuum wave functions associated with the singlet and the triplet states of the two final-state continuum electrons.

Shown in Fig. 11.3 are second-order diagrams having intermediate states in which two target electrons are excited. These diagrams correspond to corrections to the single-configuration HF description of the target atom. We use Figs. 11.3(e) and 11.3(f) to represent second-order perturbation terms which include overlap integrals between the continuum wave functions of the incident electron and one of the two outgoing electrons. Notice that all the first-order diagrams that include overlap integrals (not shown here) sum to zero because we use the general form of potential defined in Eq. (11.8).

\subsubsection{Triply differential cross section for the $\theta_{12}=\pi$ case}

For the special case treated in this chapter of $\hat{\mathbf{k}}_{1}=-\hat{\mathbf{k}}_{2}$, the differential cross section in Eq. (11.3) can be reduced to a simple form in which geometrical and dynamical dependences are clearly separated.

Once the wave functions $\Psi_{i}^{+}$and $\Phi_{f}^{-}$in Eq. (11.3) are expanded using the antisymmetrized, $L S$-coupled wave functions $\Psi_{i}$ and $\Phi_{f}$, Eq. (11.3) 


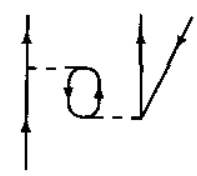

(a)

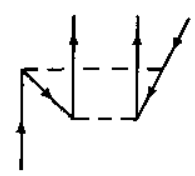

(d)

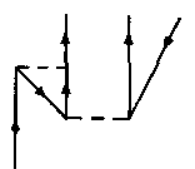

(b)

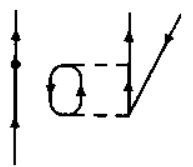

(e)

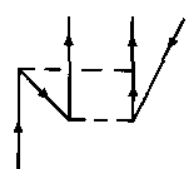

(c)

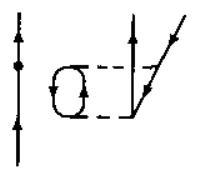

(f)

Fig. 11.3. Some other second-order diagrams contributing to $\left\langle\Phi_{f}|\Delta H| \Psi_{i}\right\rangle$. These diagrams have intermediate states in which two target electrons are excited; they correct the single-configuration Hartree-Fock description of the target atom. Diagrams (e) and (f) are used here to represent second-order terms which include overlap integrals (denoted by solid dots).

can be simplified by using the relation

$$
\begin{gathered}
\sum_{m_{1}, m_{2}} Y_{\ell_{1} m_{1}}^{*}\left(\hat{\mathbf{k}}_{1}\right) Y_{\ell_{2} m_{2}}^{*}\left(-\hat{\mathbf{k}}_{1}\right)\left\langle L M \mid \ell_{1} m_{1} \ell_{2} m_{2}\right\rangle= \\
(-1)^{\ell_{1}}\left(\frac{\left[\ell_{1}\right]\left[\ell_{2}\right]}{4 \pi}\right)^{1 / 2} Y_{L M}^{*}\left(\hat{\mathbf{k}}_{1}\right)\left(\begin{array}{ccc}
\ell_{1} & \ell_{2} & L \\
0 & 0 & 0
\end{array}\right),
\end{gathered}
$$

where the symbol $[x]$ is defined by $[x] \equiv 2 x+1$. The resulting expression is then summed over the final-state magnetic quantum numbers $m_{s_{1}}$, $m_{s_{2}}, M_{S_{\mathcal{C}}}$, and $M_{C}$ and averaged over the initial-state magnetic quantum numbers $M_{0}, M_{S_{0}}$, and $m_{s}$, i.e.,

$$
\sigma^{(3)}=\left(2\left[L_{0}\right]\left[S_{0}\right]\right)^{-1} \sum_{M_{0}, M_{S_{0}}, m_{s}} \sum_{m_{s_{1}}, m_{s_{2}}, M_{S_{C}}, M_{C}} \frac{d^{3} \sigma}{d \varepsilon_{1} \frac{d \Omega_{1} d \Omega_{2}}{2}} .
$$

The result is $[464](b)$

$$
\begin{aligned}
\sigma^{(3)}= & \frac{(-1)^{L_{0}+L_{C}} \pi}{4 k^{2}\left[L_{o}\right]} \sum_{\begin{array}{c}
L_{T}, L_{T}^{\prime}, L, L^{\prime} \\
\ell, \ell^{\prime}, S_{T}, S
\end{array}} A\left(\ell, L, S, L_{T}, S_{T}\right) A^{*}\left(\ell^{\prime}, L^{\prime}, S, L_{T}^{\prime}, S_{T}\right) \\
& \times \sum_{\lambda}[\lambda] P_{\lambda}\left(\hat{\mathbf{k}}_{1} \cdot \hat{\mathbf{k}}\right)\left[L_{T}\right]\left[L_{T}^{\prime}\right]\left([L]\left[L^{\prime}\right][\ell]\left[\ell^{\prime}\right]\right)^{1 / 2} \\
& \times\left(\begin{array}{ccc}
L & L^{\prime} & \lambda \\
0 & 0 & 0
\end{array}\right)\left(\begin{array}{ccc}
\ell & \ell^{\prime} & \lambda \\
0 & 0 & 0
\end{array}\right)\left\{\begin{array}{ccc}
L_{T} & L_{T}^{\prime} & \lambda \\
L^{\prime} & L & L_{C}
\end{array}\right\}\left\{\begin{array}{ccc}
L_{T} & L_{T}^{\prime} & \lambda \\
\ell^{\prime} & \ell & L_{0}
\end{array}\right\} .
\end{aligned}
$$


In Eq. (11.16), the amplitudes $A$ are defined by

$$
\begin{aligned}
A\left(\ell, L, S, L_{T}, S_{T}\right)= & \left(\frac{\left[S_{T}\right]}{2\left[S_{0}\right]}\right)^{\frac{1}{2}} \sum_{\ell_{1}, \ell_{2}}(-1)^{\ell_{1}+L} f\left(\ell, \ell_{1}, \ell_{2}\right)\left(\left[\ell_{1}\right]\left[\ell_{2}\right]\right)^{1 / 2} \\
& \times\left(\begin{array}{ccc}
\ell_{1} & \ell_{2} & L \\
0 & 0 & 0
\end{array}\right)\left\langle\Phi_{f}|\Delta H| \Psi_{i}\right\rangle
\end{aligned}
$$

where

$$
f\left(\ell, \ell_{1}, \ell_{2}\right)=i^{\ell+\ell_{1}+\ell_{2}} \exp \left[i\left(\delta_{\ell}+\delta_{\ell_{1}}^{C}+\delta_{\ell_{1}}+\delta_{\ell_{2}}^{C}+\delta_{\ell_{2}}\right)\right] .
$$

In Eq. (11.18), the initial-state phase shift $\delta_{\ell}$ depends on the state of the target atom and the angular momentum coupling between the incident electron and the target atom, and in general, the final-state phase shifts $\delta_{\ell_{1}}$ and $\delta_{\ell_{2}}$ depend on the state of the residual ion, the coupling between the two continuum electrons, and the coupling between the residual ion and the two-electron partial-wave state.

We now discuss the relatively simple cases of $\mathrm{H}$ and $\mathrm{He}$ targets. For the case of $\mathrm{H}, L_{0}=L_{C}=S_{C}=0, L=\ell=L_{T}, L^{\prime}=\ell^{\prime}=L_{T}^{\prime}$, and $S_{T}=S$ in Eqs. (11.16) and (11.17). For the case of He, $L_{0}=S_{0}=L_{C}=0$, $L=\ell=L_{T}, L^{\prime}=\ell^{\prime}=L_{T}^{\prime}$, and $S_{T}=S_{C}=1 / 2$. For these cases Eq. (11.16) becomes

$$
\sigma^{(3)}=\frac{\pi}{4 k^{2}} \sum_{L, L^{\prime}} \sum_{S} A(L S) A^{*}\left(L^{\prime} S\right) \sum_{\lambda}[\lambda] P_{\lambda}\left(\hat{\mathbf{k}}_{1} \cdot \hat{\mathbf{k}}\right)[L]\left[L^{\prime}\right]\left(\begin{array}{ccc}
L & L^{\prime} & \lambda \\
0 & 0 & 0
\end{array}\right)^{2} .
$$

For the lowest-order (first-order) calculations, the explicit form for the amplitude $A$ in Eq. (11.19) is

$$
\begin{aligned}
A(L S) & =C \delta_{\ell, L} 2^{-1}[S]^{1 / 2} \sum_{\ell_{1}, \ell_{2}}(-1)^{\ell_{1}} f\left(\ell, \ell_{1}, \ell_{2}\right)\left[\ell_{1}\right]\left[\ell_{2}\right]\left(\begin{array}{ccc}
\ell_{1} & \ell_{2} & \ell \\
0 & 0 & 0
\end{array}\right)^{2} \\
& \times\left\{\left[\ell_{1}\right]^{-1} R^{\ell_{1}}\left(\varepsilon_{1} \ell_{1}, \varepsilon_{2} \ell_{2} ; 1 s, \varepsilon \ell\right)+(-1)^{S}\left[\ell_{2}\right]^{-1} R^{\ell_{2}}\left(\varepsilon_{2} \ell_{2}, \varepsilon_{1} \ell_{1} ; 1 s, \varepsilon \ell\right)\right\},
\end{aligned}
$$

where $C=1$ for $\mathrm{H}$ and $C=(-1)^{S+1} \sqrt{2}$ for He. In Eq. (11.20) $R^{\ell_{1}}$ and $R^{\ell_{2}}$ are Slater integrals defined by

$$
R^{\dot{i}}(1,2 ; 3,4)=\iint P_{1}(r) P_{2}\left(r^{\prime}\right) \frac{r_{<}^{\lambda}}{r_{>}^{\lambda+1}} P_{3}(r) P_{4}\left(r^{\prime}\right) d r d r^{\prime},
$$

where $r_{<}$and $r>$ are respectively the smaller and greater of $r$ and $r^{\prime}$.

For convenience we may rewrite the triply differential cross section $\sigma^{(3)}$ in Eq. (11.16) in terms of a doubly differential cross section $\sigma^{(2)}$ and asymmetry parameters $\beta_{\lambda}$. Let $\hat{\mathbf{k}}=\hat{\mathbf{z}}$ and write $\hat{\mathbf{k}}_{1} \cdot \hat{\mathbf{k}}=\cos \theta_{1}$. Then $\sigma^{(2)}$ 
is defined by

$$
\sigma^{(2)}=\int_{0}^{2 \pi} d \phi_{1} \int_{-1}^{+1} \sigma^{(3)} d\left(\cos \theta_{1}\right)
$$

and the parameters $\beta_{\lambda}$ are determined by comparing with Eq. (11.16) the equation

$$
\sigma^{(3)}=\frac{\sigma^{(2)}}{4 \pi}\left[1+\sum_{i \geq 1} \beta_{\lambda} P_{\lambda}\left(\cos \theta_{1}\right)\right] .
$$

We use the expression in the brackets on the right hand side of Eq. (11.23) to define the relative triply differential cross section, $4 \pi \sigma^{(3)} / \sigma^{(2)}$.

\subsection{Results and discussion}

In this section, we discuss our theoretical results for the triply differential cross section (TDCS) for low-energy electron-impact ionization of atoms in the $\theta_{12}=\pi$ (i.e., $\hat{\mathbf{k}}_{1}=-\hat{\mathbf{k}}_{2}$ ) geometry. That is, the TDCS for cases in which the two final-state continuum electrons leave in opposite directions.

Figure 11.4 shows the theoretical ${ }^{[436],[464](a, b),[465]}$ and experimental ${ }^{[432,436]}$ relative TDCS for $\mathrm{H}$ for $2 \mathrm{eV}$ excess energy shared equally by the two outgoing electrons. The U-shaped angular distribution for $\mathrm{H}$ was first reported by Schlemmer et al. in 1989 for equal-sharing of $4 \mathrm{eV}$ excess energy. ${ }^{[431]}$ The overall agreement between the three sets of theoretical results and the experimental results of Rösel et al. ${ }^{[432]}$ is very good.

Notice that because these relative experimental results have been fitted to the calculated results of Pan and Starace, ${ }^{[464](a, b)}$ the comparisons with the theoretical calculations of Brauner et al. ${ }^{[436]}$ and Jones, Madison, and Srivastava ${ }^{[465]}$ are shown at a disadvantage. Although in Fig. 11.4 the three sets of theoretical results more or less agree qualitatively for the relative TDCS, there are significant quantitative discrepancies for the absolute values. The results of Brauner et al. were calculated according to Eq. (11.1) using an asymptotic wave function, which satisfies the exact asymptotic three-body boundary conditions, for $\Psi_{f}^{-}$and using a plane wave for $\Phi_{i}^{+}$. ${ }^{[436]}$ Their results are about two orders of magnitude smaller than those of the other two calculations, and they have attributed the small values of their absolute results near threshold to the wavefunction normalization they used. The other two sets of results are of the same order of magnitude. Jones, Madison, and Srivastava also used effective screening potentials to approximate the interaction between the two outgoing electrons, but they used a different ansatz to choose the effective charges. ${ }^{[465]}$ They did not include the effects due to exchange between 


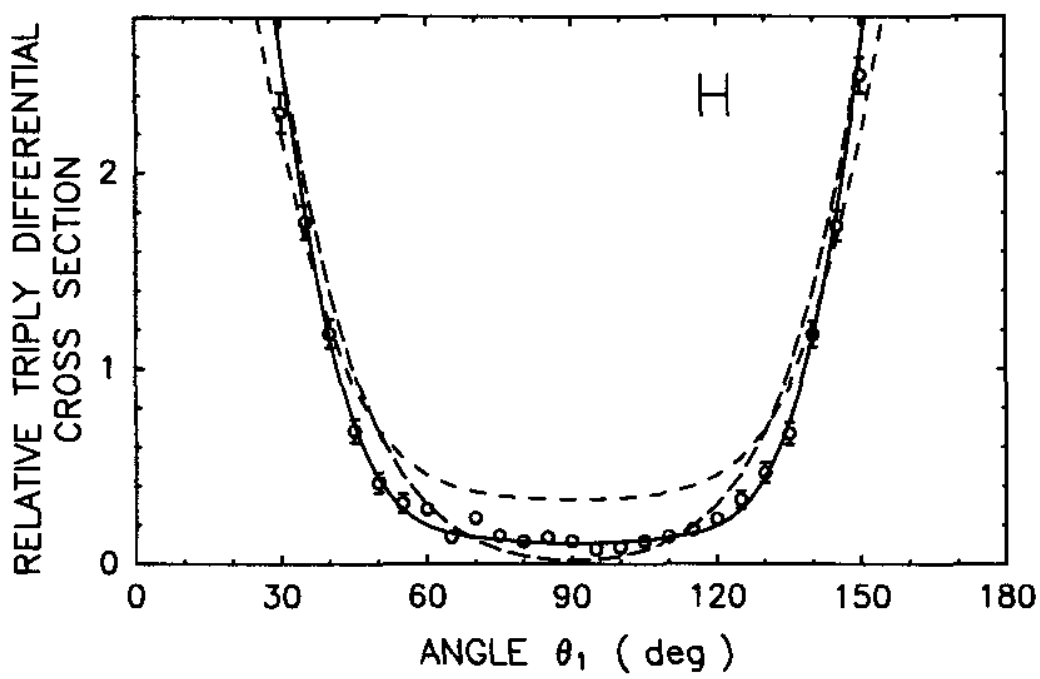

Fig. 11.4. Relative triply differential $(e, 2 e)$ cross sections for a $\mathrm{H}$-atom target for final states having $\theta_{12}=\pi$ and the continuum electrons sharing $2 \mathrm{eV}$ excess energy equally. Open circles: experimental results of Rösel et al. Ref. [432]. Curves are theoretical results. Solid curve: Pan and Starace, Ref. [464](b). Longbroken curve: Brauner et al., Ref. [436]. Short-broken curve: Jones, Madison, and Srivastava, Ref. [465]. This figure is reproduced from Ref. [464](b).

the incident electron and the target electron. The first-order calculations of Pan and Starace [464](a,b) include the diagrams of Figs. 11.1(a)-11.1(d).

Figure 11.5 shows the theoretical [439],[449],[464](a,b),[465] and experimental $[438,439]$ TDCS for $\mathrm{He}$ for $2 \mathrm{eV}$ excess energy shared equally by the two outgoing electrons. The $\mathrm{W}$-shaped angular distribution in the $\theta_{12}=\pi$ geometry for $\mathrm{He}$ for low excess energies was revealed in the measurements of Schubert et al. in $1981^{[460]}$ and of Fournier-Lagarde, Mazeau, and Huetz in $1984^{\text {[461] }}$ and was discussed in detail by Selles, Huetz, and Mazeau in 1987. [430] The first measurement for the absolute TDCS at near-threshold excess energies was reported by Rösel et al. in 1992 for He. ${ }^{[438,439]}$ The theoretical results of Pan and Starace [464](b) agree very well with the absolute results of Rösel et al. ${ }^{[438,439]}$ Those of Jones and Madison given in Ref. [439] also agree reasonably well, although they are somewhat lower than experiment near $\theta_{1}=90^{\circ}$. The main differences ${ }^{[439]}$ of these two calculations are that Jones and Madison use a local approximation to the exchange interactions compared to an exact treatment of exchange in Ref. [464]; also, the two calculations employ different values for the effective screening charges describing the interaction between the final-state continuum electrons. The earlier calculation of Jones, Madison, and Srivastava ${ }^{[465]}$ did not include any effects 


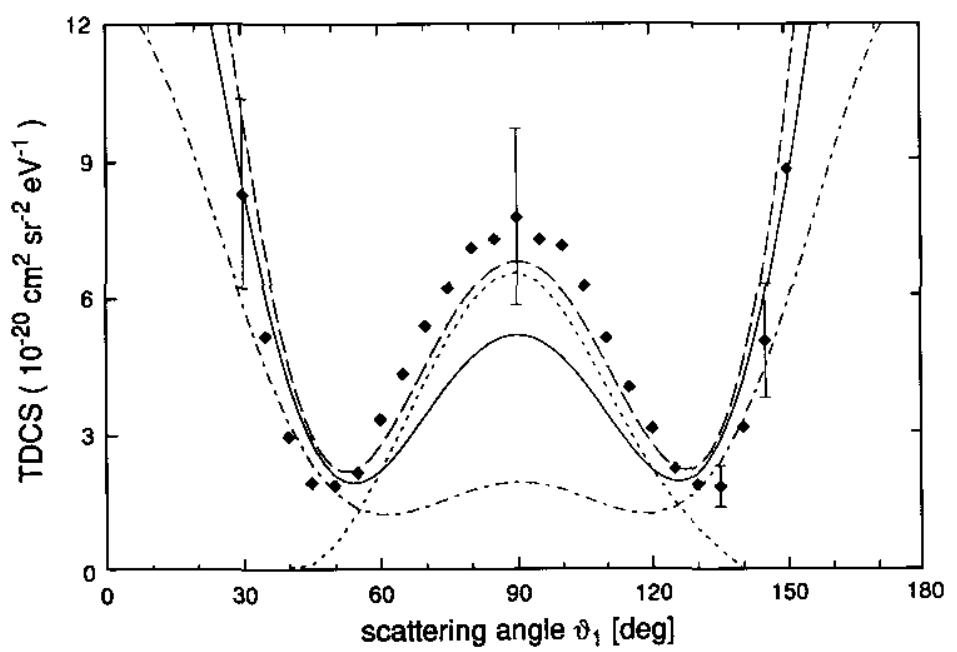

Fig. 11.5. Triply differential $(e, 2 e)$ cross sections for a He-atom target for final states having $\theta_{12}=\pi$ and the continuum electrons sharing $2 \mathrm{eV}$ excess energy equally. Solid diamonds: experimental results of Rösel et al., Ref. [438]. Curves are theoretical results. Solid curve: Jones and Madison, given in Ref. [439]. Broken curve: Pan and Starace, Ref. [464](b). Dotted curve: Crothers, Ref. [449]. Broken and dotted curve: Jones, Madison, and Srivastava, Ref. [465]. This figure is reproduced from Ref. [439].

due to the exchange interactions between the continuum electrons and the bound target electrons; their results do not show a prominent peak at $\theta_{1}=90^{\circ}$. The theoretical results of Crothers ${ }^{[449]}$ did not include contributions from the triplet states of the two outgoing electrons. However, contributions from these triplet states are zero at $\theta_{1}=90^{\circ}$, and his results agree well with the experimental results for the peak centered at $90^{\circ}$.

The striking difference between the low-energy angular distributions for $\mathrm{H}$ and $\mathrm{He}$ (shown respectively in Figs. 11.4 and 11.5) is due to short-range interactions since the asymptotic conditions are the same for both cases. As is shown in the calculations done by Pan and Starace, ${ }^{[464](a, b)}$ by Jones, Madison, and Srivastava, ${ }^{[465]}$ and by Jones and Madison, ${ }^{[439]}$ the difference can be explained in part by including the effects of the charge distributions of the target atom and the residual ion on the continuum electrons. Inclusion in addition of the effects due to exchange of the continuum electron and the bound target electrons leads then to good agreement with experiment for both cases. $[439,464](\mathrm{a}, \mathrm{b})$

According to the WPR theory, in the limit of zero excess energy, the angular distribution for the $\theta_{12}=\pi$ case is independent of the excess energy and is almost independent of the partition of the excess 
energy. [443],[444](b),[450] In 1981, Schubert et al. ${ }^{[460]}$ concluded from their measurements that at $6 \mathrm{eV}$ excess energy, the angular distribution for He was still approximately independent of the partition of the excess energy. The experimental results reported by Schlemmer et al. ${ }^{[431]}$ and by Rösel et al. ${ }^{[432]}$ have shown that the angular distribution for $\mathrm{H}$ is approximately independent of either the excess energy or its partition up to $4 \mathrm{eV}$ above threshold. Figure 11.6(a) shows the theoretical ${ }^{[464](a)}$ and experimental [431] TDCS for $\mathrm{H}$ for $4 \mathrm{eV}$ excess energy with the partition $\varepsilon_{1} / \varepsilon_{2}=7$. Since $\varepsilon_{1} \neq \varepsilon_{2}$, the TDCS is no longer symmetric about $\theta_{1}=90^{\circ} .{ }^{[464]}(\mathrm{a}, \mathrm{b})$ However, the overall difference between the results shown in Figs. 11.6(a) and 11.4 is not large. Figure 11.6(b) shows the theoretical [464](a) and experimental [431] TDCS for $\mathrm{He}$ for $4 \mathrm{eV}$ excess energy with the partition $\varepsilon_{1} / \varepsilon_{2}=7$. Similarly to the corresponding results for $\mathrm{H}$, the TDCS is no longer symmetric about $\theta_{1}=90^{\circ}$, but the overall difference between the results shown in Figs. 11.6(b) and 11.5 is not large.

Figure 11.7 compares our present theoretical results for the TDCS including electron-correlation effects with those of our first-order calculation (Ref. [464](b)) and the results of experimental measurements. [430, 438, 439] Starting from the first-order calculation, which includes effectively the diagrams of Figs. 11.1(a)-11.1(d), we first add the inter-channel interaction between the singlet and triplet states of the two outgoing electrons induced by the $\mathrm{He}^{+} 1 s$ core, i.e., the interaction described by the matrix element $\left\langle 1 s\left[\varepsilon_{1} \ell_{1} \varepsilon_{2} \ell_{2}\left({ }^{1} L\right)\right]\left({ }^{2} L\right)\left|\sum r_{i j}^{-1}\right| 1 s\left[\varepsilon_{1} \ell_{1} \varepsilon_{2} \ell_{2}\left({ }^{3} L\right)\right]\left({ }^{2} L\right)\right\rangle$. With the interchannel interaction added, all the diagrams in Fig. 11.2 are now included. Then, we include the diagrams of the type shown in Fig. 11.1(e) and, using the approximation discussed in Sec. 11.2.2, the diagrams of the types shown in Figs. 11.1(f) and 11.1(g). The diagram of Fig. 11.1(e) is found to have a relatively small effect as compared to the other two diagrams in Figs. 11.1(f) and 11.1(g). Finally, we include the second-order diagrams in Fig. 11.3, which correspond to corrections to the single-configuration $\mathrm{HF}$ description for the wave function of the He atom. Note that except for the diagrams involving overlap integrals (which are found to be relatively small for the present case), these corrections have been treated previously by Pan and Starace. ${ }^{[464](b)}$ One can see in Fig. 11.7 that the overall change in the TDCS is not very large after including these electron-correlation effects. The theoretical results are improved appreciably near $\theta_{1}=50^{\circ}$ and $130^{\circ}$, where the TDCS displays minima, although the peak at $\theta_{1}=90^{\circ}$ becomes somewhat lower. The effect of the inter-channel interaction is relatively strong.

In 1990, Selles, Mazeau, and Huetz reported measurements of TDCS for $\mathrm{Ne}, \mathrm{Ar}$, and $\mathrm{Kr}$ targets for equal-sharing of $0.5,1,2$, and $4 \mathrm{eV}$ excess energies. ${ }^{435]}$ These results were compared with the results for $\mathrm{He}$, 

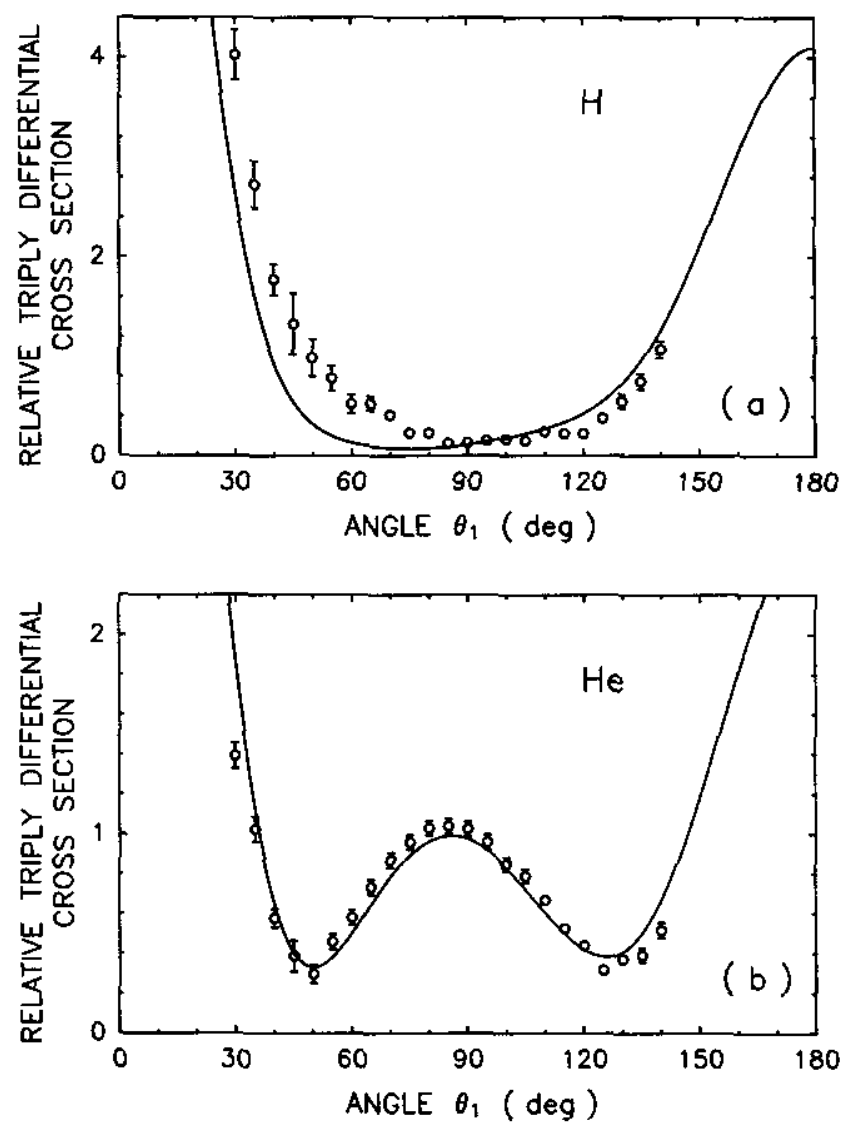

Fig. 11.6. Relative triply differential $(e, 2 e)$ cross sections for (a) $\mathrm{H}$ and (b) He targets for final states having $\theta_{12}=\pi$ and the continuum electrons having $1 / 2 k_{1}^{2}=3.5 \mathrm{eV}$ and $1 / 2 k_{2}^{2}=0.5 \mathrm{eV}$. Open circles: experimental results of Schlemmer et al., Ref. [431]. Solid curves: theoretical results of Pan and Starace, Ref. [464](a). This figure is reproduced from Ref. [464](a).

and the angular distributions for all four of these targets were shown to become stable as the excess energy was lowered, in agreement with the prediction of the WPR theory. [443],[444](b),[450] Nevertheless, the upper limits of the excess energy below which the angular distribution curves are stable are different for these targets. The upper limit is found to change from about $2 \mathrm{eV}$ to below $1 \mathrm{eV}$ when following the $\mathrm{He}$ to $\mathrm{Kr}$ chain. This is presumably due to the stronger electron-correlation effects in the heavier target atoms. Rösel et al. also measured the TDCS for $\mathrm{Kr}$ and $\mathrm{Xe}$ targets for equal-sharing of $2 \mathrm{eV}$ excess energy. ${ }^{[432]}$ The results for these heavy rare-gas targets also show strong target dependence. Pan and Starace ${ }^{[464](b)}$ have performed first-order calculations for these targets; 


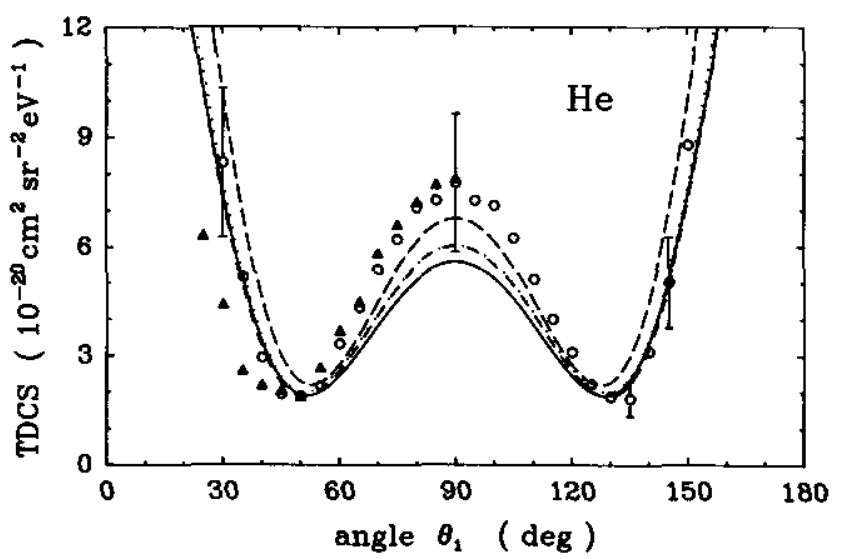

Fig. 11.7. Triply differential $(e, 2 e)$ cross sections for a He-atom target for final states having $\theta_{12}=\pi$ and the continuum electrons sharing $2 \mathrm{eV}$ excess energy equally. Open circles: absolute experimental results of Rösel et al., Ref. [438]. Solid triangles: relative experimental results of Selles, Huetz, and Mazeau, Ref. [430], fitted to the result of Rösel et al. at $\theta_{1}=50^{\circ}$. Curves are the present theoretical results. Broken curve: first-order results for the case in which the diagrams of Figs. 11.1(b)-11.1(d) sum to zero. Dotted curve: results also including the final-state inter-channel interaction. Broken-and-dotted curve: results also including the diagrams of Figs. $11.1(\mathrm{e})-11.1(\mathrm{~g})$. Solid curve: results also including all the diagrams in Fig. 11.3.

for the most part, the calculated and the measured results for $\mathrm{Ne}, \mathrm{Ar}, \mathrm{Kr}$, and $\mathrm{Xe}$ agree qualitatively. However, the comparison indicates the need in future calculations to include electron-correlation effects. Figures 11.8(a, b) show the theoretical ${ }^{[464](b)}$ and experimental ${ }^{[435]}$ TDCS for $\mathrm{Ne}$ and Ar targets for equal-sharing of $0.5 \mathrm{eV}$ excess energy, which is the lowest excess energy used in the measurements. One can see a rough qualitative agreement between theory and experiment. However, the calculated results for $\mathrm{Kr}$ do not agree with the measured ones at $0.5 \mathrm{eV}$ excess energy. ${ }^{[464](b)}$ Figures 11.9(a) and 11.9(b) show the theoretical ${ }^{[464](b)}$ and experimental [432,435] TDCS for $\mathrm{Kr}$ and Xe for equal-sharing of $2 \mathrm{eV}$ excess energy. Good agreement between the calculated and the measured results for Xe can be seen for this excess energy.

The $\mathrm{H}$ atom and the He atom are the two simplest target atoms, and the theoretical explanation for the difference between the TDCS for $\mathrm{H}$ and $\mathrm{He}$ in the $\theta_{12}=\pi$ geometry turns out to be relatively simple. [464] The difference between the TDCS for either $\mathrm{H}$ or $\mathrm{He}$ and the TDCS for any other target atom is in general more complicated; the $\mathrm{Li}$ atom can be used as an example to demonstrate this point. The Li atom has an outer subshell which is isoelectronic to the $\mathrm{H}$ atom, and so Eqs. (11.19) 

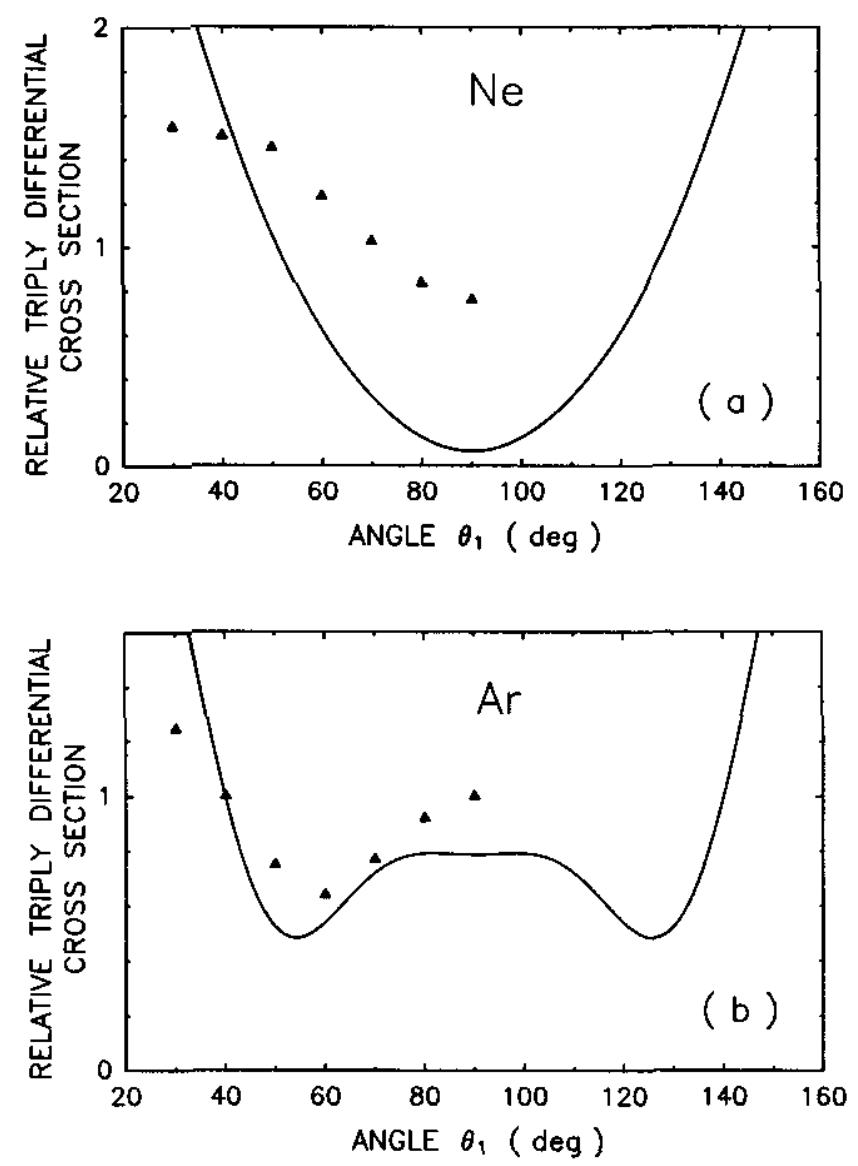

Fig. 11.8. Relative triply differential $(e, 2 e)$ cross sections for (a) $\mathrm{Ne}$ and (b) $\mathrm{Ar}$ targets for final states having $0_{12}=\pi$ and the continuum electrons sharing $0.5 \mathrm{eV}$ excess energy equally. Solid triangles: experimental results of Selles, Mazeau, and Huetz, Ref. [435]. Solid curves: theoretical results of Pan and Starace, Ref. [464](b). This figure is reproduced from Ref. [464](b).

and $(11.20)$ can be used to calculate its TDCS provided that the $1 \mathrm{~s}$ labels in Eq. (11.20) are changed to $2 s$ labels. Figure 11.10 shows the theoretical TDCS for $\mathrm{Li}$ for equal-sharing of $2 \mathrm{eV}$ excess energy. [464](c) One immediately sees that the angular distribution for $\mathrm{Li}$ is more complex than the ones for $\mathrm{H}$ and $\mathrm{He}$. Table 11.1 presents the parameters $\sigma^{(2)}$ and $\beta_{\lambda}$ that describe the angular distributions for $\mathrm{H}, \mathrm{He}$, and $\mathrm{Li}$ targets for equal-sharing of $2 \mathrm{eV}$ excess energy. [464](c) One notices that $\beta_{2}$ is the largest asymmetry parameter for $\mathrm{H}, \beta_{4}$ is the largest for $\mathrm{He}$, and both $\beta_{6}$ and $\beta_{8}$ are very large for Li.

Table 11.2 compares the relative magnitudes and phases of the partial- 

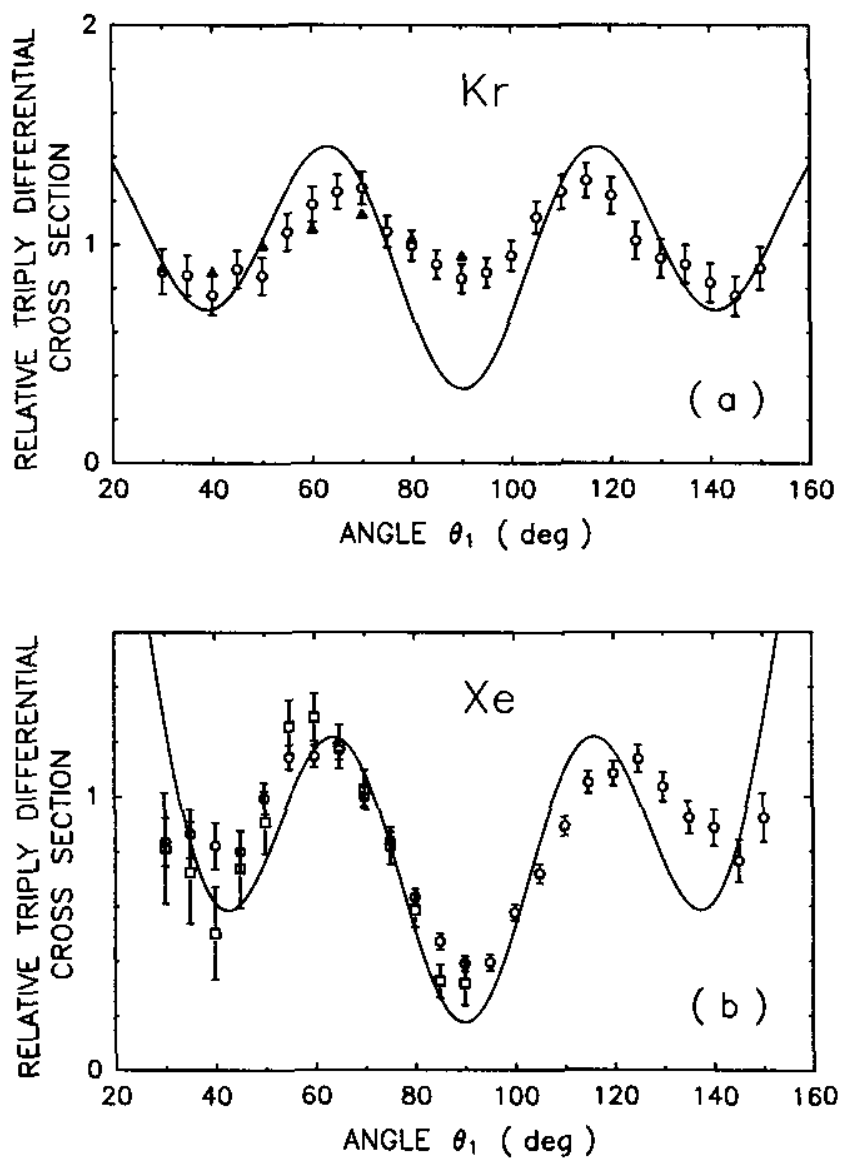

Fig. 11.9. Relative triply differential $(e, 2 e)$ cross sections for (a) $\mathrm{Kr}$ and (b) $\mathrm{Xe}$ targets for final states having $\theta_{12}=\pi$ and the continuum electrons sharing $2 \mathrm{eV}$ excess energy equally. Solid triangles: experimental results of Selles, Mazeau, and Huetz, Ref. [435]. Open circles: experimental results of Rösel et al., Ref. [432], for $2 \mathrm{eV}$ above the $\mathrm{Xe}^{+}\left({ }^{2} P_{3 / 2}\right)$ threshold. Open squares: experimental results of Rösel et al., Ref. [432], for $2 \mathrm{eV}$ above the $\mathrm{Xe}^{+}\left({ }^{2} P_{1 / 2}\right)$ threshold. Solid curves: theoretical results of Pan and Starace, Ref. [464](b). This figure is reproduced from Ref. [464](b).

wave amplitudes $A(L S)$ (cf. Eq. (11.20)) for $\mathrm{H}, \mathrm{He}$, and $\mathrm{Li}$ targets for equal-sharing of $2 \mathrm{eV}$ excess energy. ${ }^{[464](\mathrm{c})}$ One can see that the relative magnitudes of these amplitudes are very similar for $\mathrm{H}$ and $\mathrm{He}$. The arguments of these amplitudes are also very similar for $\mathrm{H}$ and $\mathrm{He}$ except for the case of the ${ }^{1} S^{e}$ amplitudes, which differ by more than $1.5 \mathrm{rad}$. This difference affects the TDCS in Eq. (11.19) primarily via the interference terms [i.e., $A\left({ }^{1} S^{e}\right) A^{*}\left({ }^{1} D^{e}\right)+A\left({ }^{1} D^{e}\right) A^{*}\left({ }^{1} S^{e}\right)$ ] between the $L=0$ and $L=2$ 
Table 11.1. Parameters $\sigma^{(2)}$ and $\beta_{i}$ determining the triply differential cross section $\sigma^{(3)}$ [cf. Eq. (11.23)] for $E_{\text {ex }}=2 \mathrm{eV}$ for $\mathrm{H}, \mathrm{He}$, and Li targets. Dashed lines indicate values smaller than 0.003 .

\begin{tabular}{cccc}
\hline Parameter & $\mathrm{H}^{a}$ & $\mathrm{He}^{b}$ & $\mathrm{Li}^{c}$ \\
\hline$\sigma^{(2)}$ (a.u.) & 3.73 & 0.781 & 34.5 \\
$\beta_{2}$ & 3.090 & 1.082 & 1.638 \\
$\beta_{4}$ & 2.367 & 1.868 & 0.856 \\
$\beta_{6}$ & 0.855 & 0.338 & 1.881 \\
$\beta_{8}$ & 0.115 & 0.023 & 2.036 \\
$\beta_{10}$ & 0.008 & & 0.924 \\
$\beta_{12}$ & & & 0.216 \\
$\beta_{14}$ & & & 0.029 \\
\hline \hline
\end{tabular}

${ }^{a}$ Ref. [464](b), Table III.

${ }^{b}$ First-order results corresponding to the long-dashed curves in Figs. 11.5 and 11.7.

${ }^{c}$ Ref. [464](c), Table I.

partial waves, which contribute to the asymmetry parameter $\beta_{2}$. The interference terms for $\mathrm{H}$ and for $\mathrm{He}$ largely explain the observed difference between the angular distributions for the two targets. ${ }^{[464] \text { (a) }}$ The difference between the phases of the ${ }^{1} S^{e}$ amplitudes for the two targets stems mainly from the differences between the one-electron s-wave phase shifts for the corresponding continuum electrons in the two cases due to short-range interactions.

While for $\mathrm{H}$ and $\mathrm{He}$ targets the phase shifts of the $\ell \geq 1$ partial waves of the continuum electrons are all close to zero, this is no longer true for heavier targets. Also, for heavier targets, the ejected electron in the low-energy ionization process is not the $1 \mathrm{~s}$ electron. As a result, more partial-wave amplitudes for the two outgoing electrons are important in describing the TDCS than for $\mathrm{H}$ and $\mathrm{He}$ targets. One can see in Table 11.2 that six partial-wave amplitudes for Li are significant, but only four amplitudes for $\mathrm{H}$ and $\mathrm{He}$ are significant.

It is shown in Fig. 11.5 for He that when $\theta_{12}=\pi, \theta_{1}=90^{\circ}$, and $E_{\mathrm{ex}}=$ $2 \mathrm{eV}$, the calculations by Crothers, ${ }^{[449]}$ by Pan and Starace, ${ }^{[464](\mathrm{b})}$ and by Jones and Madison [439] all agree fairly well with the experimental TDCS. However, the threshold behaviors of the TDCS for the $\theta_{12}=\pi$ case given by the theoretical methods used in these calculations are very different. On the one hand, Crothers' results for the total electron-impact ionization cross section in the range $0 \leq E_{\mathrm{ex}} \leq 6 \mathrm{eV}$ have an energy dependence $E_{\mathrm{ex}}^{1.127}$, and his result for the TDCS for the $\theta_{12}=\pi$ case varies according 


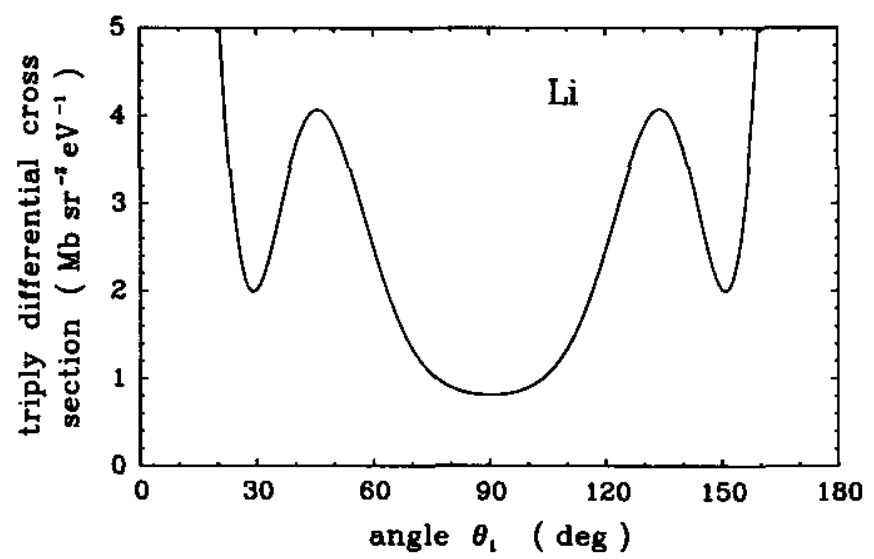

Fig. 11.10. Triply differential $(e, 2 e)$ cross sections for a Li-atom target for final states having $\theta_{12}=\pi$ and the continuum electrons sharing $2 \mathrm{eV}$ excess energy equally. Solid curve: theoretical results of Pan and Starace, Ref. [464](c). This figure is reproduced from Ref. [464](c).

Table 11.2. Relative amplitude and phase for electron-impact ionization scattering amplitudes $A(L S)$ for $\mathrm{H}, \mathrm{He}$, and $\mathrm{Li}$ targets for final-state electron kinetic energies $1 / 2 k_{1}^{2}=1 / 2 k_{2}^{2}=1 \mathrm{eV}$. Only the first six partial waves for each target are shown.

\begin{tabular}{|c|c|c|c|c|c|c|}
\hline \multirow{2}{*}{$\begin{array}{l}\text { Partial wave } \\
\qquad 2 S+1 L^{\pi}\end{array}$} & \multicolumn{3}{|c|}{ Relative amplitude $|A(L S)| /\left|A\left({ }^{1} S^{e}\right)\right|$} & \multicolumn{3}{|c|}{$\arg A(L S)(\mathrm{rad})$} \\
\hline & $\mathrm{H}^{a}$ & $\mathrm{He}^{b}$ & $\mathrm{Li}^{a}$ & $\mathrm{H}^{a}$ & $\mathrm{He}^{b}$ & $\mathrm{Li}^{a}$ \\
\hline${ }^{1} S^{e}$ & $1.000^{\circ}$ & $1.000^{\circ}$ & $1.000^{c}$ & 2.95 & 4.52 & 2.85 \\
\hline${ }^{3} P^{\circ}$ & 0.471 & 0.466 & 0.236 & 1.10 & 1.37 & 4.13 \\
\hline${ }^{1} D^{e}$ & 0.504 & 0.577 & 0.121 & 2.32 & 2.58 & 0.88 \\
\hline${ }^{3} F^{\circ}$ & 0.324 & 0.211 & 0.251 & 1.27 & 1.20 & 5.48 \\
\hline${ }^{1} G^{e}$ & 0.036 & 0.029 & 0.192 & 1.28 & 1.26 & 0.59 \\
\hline${ }^{3} H^{0}$ & 0.023 & 0.009 & 0.164 & 0.80 & 0.46 & 5.84 \\
\hline
\end{tabular}

${ }^{a}$ Ref. [464](c), Table II.

${ }^{b}$ First-order results corresponding to the long-dashed curves in Figs. 11.5 and 11.7.

${ }^{c}$ The values of $\left|A\left({ }^{1} S^{e}\right)\right|$ for $\mathrm{H}, \mathrm{He}$, and $\mathrm{Li}$ targets are respectively $0.3430,0.2076$, and 0.9003 .

to $E_{\mathrm{ex}}^{1.127-3 / 2}$. [449] These energy dependences agree with the WPR theory in the limit $E_{\mathrm{ex}} \rightarrow 0$. [443],[445](b) The diverging behavior of this TDCS as $E_{\text {ex }} \rightarrow 0$ is related to the rapid narrowing of the width of the distribution with respect to $\theta_{12}$ centered around $\theta_{12}=\pi$, which varies as $E_{\mathrm{ex}}^{1 / 4}$. On the other hand, the TDCS for the $\theta_{12}=\pi$ case given by calculations using the 


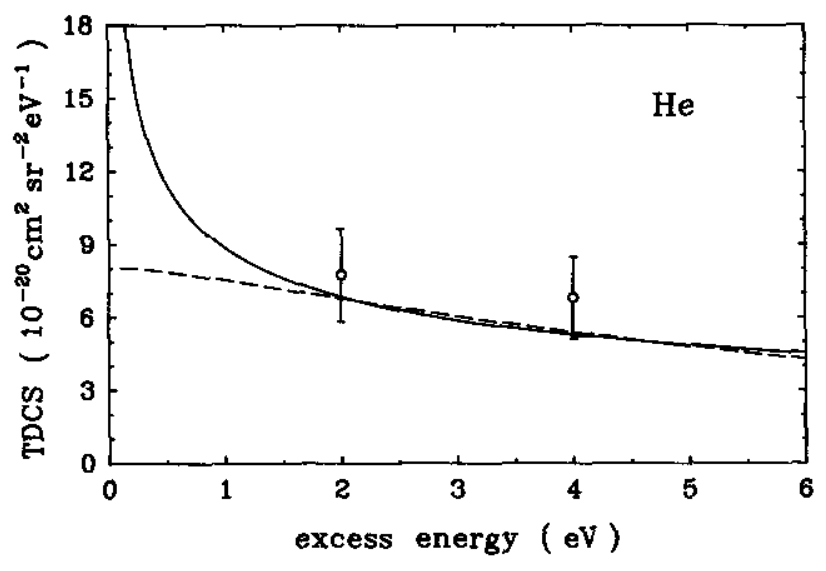

Fig. 11.11. Triply differential $(e, 2 e)$ cross sections for a He-atom target for final states having $\theta_{12}=\pi$ and $\theta_{1}=\theta_{2}=90^{\circ}$ and the continuum electrons sharing equally the excess energy in the range $0-6 \mathrm{eV}$. Open circles: experimental results of Rösel et al., Ref. [438]. Solid curve: theoretical results of Crothers, Ref. [449]. Broken curve: the first-order theoretical results of Pan and Starace.

effective-charge approximation [456]-[458],[464],[465] is independent of the excess energy in the $E_{\mathrm{ex}} \rightarrow 0$ limit. This threshold behavior can be obtained by analyzing the wave functions used in such calculations. Figure 11.11 compares the results of our first-order calculations and the results of Crothers ${ }^{[449]}$ with the measured TDCS for He for the case of $\theta_{12}=\pi$ and $\theta_{1}=90^{\circ}$ in the energy range $0 \leq E_{\mathrm{ex}} \leq 6 \mathrm{eV}$. Crothers explicitly gives the TDCS results only for 1 and $2 \mathrm{eV}$ excess energies. Here we extract results for other energies using both Fig. 1 and Eqs. (74) and (86) of Ref. [449]; the TDCS as a function of the excess energy $E_{\text {ex }}$ for the case of $\theta_{12}=\pi$ and $\theta=90^{\circ}$ is given by $8.9\left(E_{\mathrm{ex}} / \mathrm{eV}\right)^{-0.373} \times 10^{-20} \mathrm{~cm}^{2} \mathrm{sr}^{-2} \mathrm{eV}^{-1}$. The available absolute experimental results are also plotted. ${ }^{[438]}$ One sees that although the two theoretical curves agree well with the available experimental results at and above $2 \mathrm{eV}$ excess energy, they depart from each other as the excess energy decreases below $2 \mathrm{eV}$. At present, the range of validity of the WPR theory is not known. However, it is clear that as the excess energy approaches zero the absolute TDCS results calculated by using approximations employing effective screening potentials [456]-[458],[464],[465] cannot be relied upon.

\subsection{Concluding remarks}

We have discussed theoretical and experimental results for low-energy electron-impact ionization of atoms in the $\theta_{12}=\pi$ geometry. Our own 
calculations have used a distorted-wave method which incorporates effective screening potentials (with effective charges conforming to those specified by Peterkop and by Rudge and Seaton) to approximate the interaction between the final-state continuum electrons. Good agreement with experiment ${ }^{[438]}$ is obtained for the absolute triply differential cross section of $\mathrm{He}$ for an excess energy as low as $2 \mathrm{eV}$.

For $\mathrm{H}$ atom and $\mathrm{He}$ atom targets, only one-electron $\ell=0$ partial waves are substantially distorted by the target atom (and by the residual ion in the case of $\mathrm{He}$ ) and so acquire non-Coulomb phase shifts which differ significantly from zero. The different $s$-wave phase shifts for the two targets are the main causes for the substantial difference between the arguments of the ${ }^{1} S^{e}$ amplitudes, which in turn is the main reason that the interference terms between the ${ }^{1} S^{e}$ and ${ }^{1} D^{e}$ amplitudes for the two cases differ, causing thereby the $\beta_{2}$ parameters to differ. Thus the observed difference between the angular distributions for $\mathrm{H}$ and $\mathrm{He}{ }^{[430,431,432]}$ is largely explained.

For $\mathrm{H}$ and $\mathrm{He}$, inclusion of electron-correlation effects changes the angular distributions for the $\theta_{12}=\pi$ case only slightly from those obtained from first-order calculations which include distortion effects arising from both the direct and the exchange interactions. For He, comparison with experiment is improved near $\theta_{1}=50^{\circ}$ and $130^{\circ}$, where the angular distribution has minima, but is made worse near $\theta_{1}=90^{\circ}$ where there is a local maximum. Amplitudes for $L>3$ two-electron partial waves are not important for describing the angular distributions for $\mathrm{H}$ and $\mathrm{He}$, as was found empirically. ${ }^{[431]}$

In comparison to $\mathrm{H}$ and $\mathrm{He}$, in the case of heavier target atoms, [432, 435] more one-electron partial waves are substantially distorted, and consequently, more two-electron partial-wave amplitudes are important for describing the angular distributions. Also, for heavier targets electroncorrelation effects become very important. 